\title{
SÍNTESE DE POLI(UREIA-URETANO) VIA POLIMERIZAÇÃO EM MINIEMULSÃO EM REATOR MICROONDAS
}

\author{
A. VALERIO ${ }^{1}$, M. FORTUNY ${ }^{2}$, A. F. SANTOS ${ }^{3}$, P. H. H. ARAUJO ${ }^{1}$,C. SAYER ${ }^{1}$ \\ ${ }^{1}$ Universidade Federal de Santa Catarina, Departamento de Engenharia Química e Engenharia de \\ Alimentos \\ ${ }^{2}$ Núcleo de Estudos em Sistemas Coloidais, Instituto de Tecnologia e Pesquisa. \\ ${ }^{3}$ Universidade Federal do Paraná, Departamento de Engenharia Química \\ E-mail para contato: ale_valerio1@yahoo.com.br
}

RESUMO - A irradição microondas tem sido uma alternativa interessante como sistema de aquecimento em diferentes reações químicas. Neste trabalho foi realizado o estudo das reações em reator microondas em comparação ao aquecimento convencional, onde os efeitos da temperatura e potência do microondas na síntese de poli(ureia-uretano) (PU) via polimerização miniemulsão foram discutidos. O polímero final foi avaliado por FTIR e o tamanho e distribuição das partículas caracterizadas por dispersão dinâmica de luz (DLS). Em um estudo comparativo, a polimerização conduzida em reator microondas apresentou maior velocidade de reação e maior conversão quando comparado com as reações com aquecimento convencional, resultando em uma redução significativa no tempo de reação, bem como a possibilidade de controlar a formação de grupos de ureia/uretano com o uso do reator microondas na sintese de PU via polimerização em miniemulsão.

\section{INTRODUÇÃO}

Microondas é um tipo de energia radiante que faz parte do espectro eletromagnético, ou seja, é uma forma de radiação eletromagnética. As microondas são classificadas como radiação não ionizante, com efeito, estritamente térmico, tendo como princípio básico o aquecimento através da vibração molecular. Portanto, o aquecimento por microondas é um processo de aquecimento muito diferente quando comparado ao aquecimento convencional (Fortuny et al., 2008; Costa et al., 2009; Marcasuza et al., 2011; Bogdal, 2012). Durante os últimos anos, o uso de reatores microondas comerciais com excelente controle de temperatura e potência aumentou significativamente a confiabilidade das reações de microondas em diferentes áreas da pesquisa (Costa et al., 2009; Xiong et al., 2010; Melo et al., 2010; Marcasuza et al., 2011; Bogdal, 2012, Yu e Chen, 2013). Radiação microondas utilizada como método rápido e eficiente de aquecimento do meio reacional apresenta vantagens significativas sobre os métodos térmicos convencionais. Estudos mostraram que quando comparada com reações utilizando aquecimento convencional, as reações utilizando radiação microondas têm como principal vantagem taxas de reação mais elevadas, levando assim a uma maior conversão dos monômeros em um curto período de tempo (Li et al., 2007; Xiong et al., 2010; Yu e Chen, 2013). Atualmente, a 


\section{9 a 22 de outubro de 2014 \\ Florianópolis/SC}

polimerização via miniemulsão utilizando radiação microondas não é amplamente investigada havendo poucos relatos na literatura.

Em estudos realizados por Xiong et al., (2010) na obtenção do copolímero fluoroacrilato via polimerização radicalar em miniemulsão usando radiação microondas, os autores verificaram que os diâmetros médios das partículas de látex obtido via radiação microondas eram menores e mais monodispersos e apresentaram maior estabilidade quando comparado aos obtidos por meio de aquecimento convencional. Adlington et al. (2013) investigaram a síntese de oligômeros de metacrilato de metila por polimerização de transferência de cadeia controlado por ATRP (Atom Transfer Radical Polimerization) utilizando 2,2'- azobisisobutyronitrile, 2,2'-azo-bisiobutironitrila (AIBN) como catalisador mostrou que as reações foram aceleradas consideravelmente com a utilização de aquecimento por microondas. Segundo os autores a aceleração do processo de síntese durante aquecimento por microondas é atribuído ao aquecimento seletivo das espécies de radicais oligoméricas.

A obtenção de poli(ureia-uretano) via polimerização em miniemulsão tem atraído muita atenção nas últimas décadas devido ao seu uso em diferentes áreas como farmacêutica, médica e de cosméticos, principalmente como meio para libertação controlada de fármacos devido às suas excelentes propriedades físicas e de biocompatibilidade (Torini et al., 2005; Crespy et al., 2007; Zanetti-ramos et al., 2009; Ding et al., 2011; Gaudin e Sintes-zydowicz de 2011; Valerio et al., 2013). Com base no que foi apresentado, o uso de radiação microondas na polimerização via miniemulsão do diisocianato (IPDI) foi investigada, sendo os efeitos cinéticos dos parâmetros de reação, como temperatura, potência e tipo de poliol investigados, uma vez que a literatura não relata trabalhos de polimerização em miniemulsão em etapas conduzidas em reator microondas como proposto nesse trabalho.

\section{MATERIAIS E MÉTODOS}

2.1 Materiais: Para a realização das reações em miniemulsão foram utilizados os monômeros, diisocianato de isoforona (IPDI, $98 \%$, Mw 222 g/mol, Sigma), como polióis poli(etilenoglicol) (PEG), um ácido graxo monoinsaturado contendo dois grupos hidroxila, com massa molar média 380420 g/mol (PEG 400, Sigma Aldrich) e óleo de mamona (100 \%, Mw 928 g/mol, Linfar), composto rico em cadeias de ácidos graxos constituído majoritariamente por ácido ricinoléico (Shahidi, 2005). Como surfactantes, foi utilizado dodecil sulfato de sódio (SDS) adquirido a partir da Sigma-Aldrich. Na quantificação de grupamentos NCO livres ao final das reações os reagentes n-dibutilamina (99\%, Vetec), tolueno (99,5\%, Vetec), propanol (99,5\%, Synth), acido clorídrico $1 \mathrm{~N}$ ( $\mathrm{HCl}$, Cronoline, P.A) e azul de bromofenol $0.1 \%$ (Lafan) foram utilizados. Todos os reagentes foram utilizados como recebidos sem previa purificação.

\subsection{Métodos: Polimerização por aquecimento convencional}

As nanopartículas de PU foram sintetizadas via polimerização em miniemulsão de acordo com o procedimento descrito a seguir. A fase aquosa (90\% em massa) foi preparada com $10 \%$ de surfactante SDS em relação à fase orgânica. A fase orgânica foi preparada com $10 \%$ 
PEG 400 (em relação a monômeros), IPDI e óleo de mamona em uma razão molar NCO:OH mantida constante em todas as reações em 1,5:1. A fase aquosa foi adicionada lentamente à fase orgânica e mantida sob agitação magnética por $5 \mathrm{~min}$ a $40{ }^{\circ} \mathrm{C}$. A miniemulsão foi então preparada pela sonificação da emulsão anterior durante 300 s a uma amplitude de $20 \%$ (Malvern - Hidro 2000MU) utilizando banho de gelo na tentativa de retardar o início da reação. Em seguida a temperatura foi aumentada para $70{ }^{\circ} \mathrm{C}$ (salvo menção em contrário) e a reação mantida em reator de vidro encamisado conectado a um banho termostático com circulação de água por 5 horas a baixa pressão (1 atm).

\subsection{Polimerização em reator microondas}

As reações de microondas foram realizadas em reator microondas Discover S-Class, CEM localizado no Laboratório Núcleo de Estudos de Sistemas Coloidais - NUESC/UNIT. Para comparar os resultados, a miniemulsão inicial foi obtida utilizando a mesma metodologia descrita acima, para aquecimento convencional. No entanto, após a sonificação a miniemulsão foi colocada em reator microondas utilizando balão de vidro específico para uso em microondas com capacidade de $35 \mathrm{~mL}$. A polimerização se deu pela aplicação de radiação microondas a uma potência de no máximo $50 \mathrm{~W}, 100 \mathrm{~W}, 150 \mathrm{~W}$ ou $300 \mathrm{~W}$ até a temperatura desejada $\left(50{ }^{\circ} \mathrm{C}\right.$ a 80 $\left.{ }^{\circ} \mathrm{C}\right)$ ser alcançada. As reações foram mantidas por 5 horas no recipiente microondas. Para evitar o aquecimento não uniforme durante a polimerização em microondas, o reator foi equipado com uma placa eletromagnética localizada abaixo da cavidade do microondas, que permitiu a agitação das amostras durante as reações. O equipamento faz uso de um sensor infravermelho, sem contato, para medidas de temperatura. Ao final de cada reação um jato de ar no interior da cavidade do sistema foi utilizado para reduzir instantaneamente a temperatura da miniemulsão final. Durante as reações foram retiradas amostras em duplicatas em intervalos regulares para caracterização e a conversão do monômero IPDI foi determinada a partir da titulação de volta dos grupamentos NCO livres seguindo metodologia descrita pela ASTM D-2572.

2.3 Métodos: Caracterização do polímero: Os produtos formados na reação foram verificados por espectroscopia de infravermelho com transformada de Fourier (FTIR), em modo de refletância total atenuada (ATR). A banda de absorção com localização do pico em $2272 \mathrm{~cm}^{-1}$, devido a $\mathrm{N}=\mathrm{C}=\mathrm{O}$ dos grupos isocianato, foi definida para identificação do IPDI. Para a identificação dos produtos de reação presentes no polímero final à banda de absorção localizada entre 1680-1650 $\mathrm{cm}^{-1}$ referente ao grupo N-H e de ureia e a banda de absorção entre 1740-1700 $\mathrm{cm}^{-1}$, atribuída a vibração do grupamento $\mathrm{C}=\mathrm{O}$ do uretano foram avaliadas. $\mathrm{O}$ diâmetro médio das nanopartículas de PU foi avaliado por dispersão dinâmica de luz (DLS, Zetasizer Nano S, de Malvern). As amostras foram preparadas pela diluição do látex final (1:10) em água deionizada, as medidas foram tomadas em duplicata, a $25^{\circ} \mathrm{C}$. 


\section{RESULTADOS E DISCUSSÃO}

\subsection{Comparação entre polimerização em microondas e aquecimento convencional: Influência da temperatura na polimerização em miniemulsão}

Visando verificar a influência da temperatura nas reações de polimerização em miniemulsão, foram realizadas reações variando a temperatura na faixa de $50{ }^{\circ} \mathrm{C}$ até $80{ }^{\circ} \mathrm{C}$ usando aquecimento convencional e microondas com potência de $150 \mathrm{~W}$. Os resultados em termos de conversão e tamanho de partículas são apresentados na Figura 1.

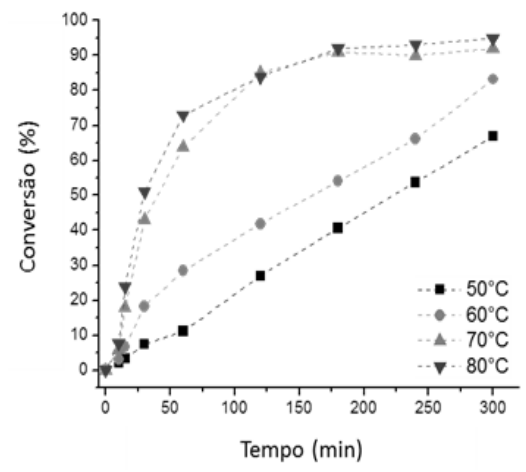

(a)

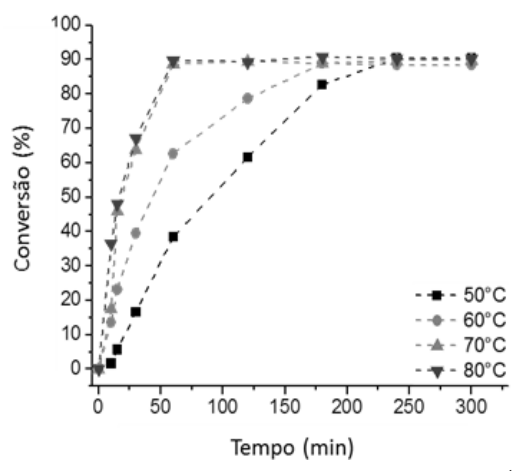

(c)

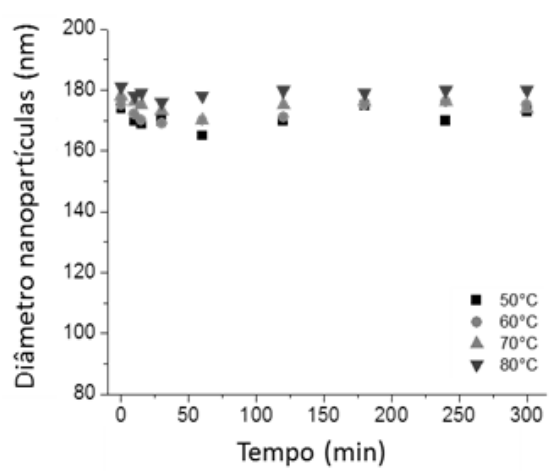

(b)

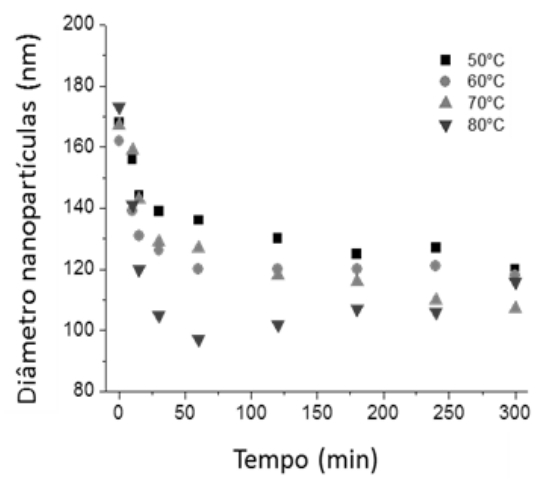

(d)

Figura 1 - Evolução da conversão (a e c) e diâmetro das nanopartículas (b e d) da PU durante polimerização em miniemulsão com aquecimento convencional (a e b) e radiação microondas (c e d) sintetizadas utilizando óleo de mamona/PEG400 como poliol.

Em ambos os casos, a taxa de reação aumentou quando a temperatura da reação foi aumentada de $50^{\circ} \mathrm{C}$ para $60{ }^{\circ} \mathrm{C}$ e de $60{ }^{\circ} \mathrm{C}$ para $70{ }^{\circ} \mathrm{C}$. No entanto, um aumento adicional de temperatura de $70{ }^{\circ} \mathrm{C}$ e $80^{\circ} \mathrm{C}$, para ambos os sistemas, resultou apenas em um ligeiro aumento na taxa de reação. Isto é atribuído ao aumento das reações com os grupos $\mathrm{OH}$ da água, com formação de ureia, o que afeta a estequiometria da reação e, assim, diminui ligeiramente a velocidade da reação, contrabalançando o efeito da temperatura mais elevada, como observado por Valério et al. (2013) para reações com aquecimento convencional. Por outro lado, o efeito da 
energia de microondas na evolução da conversão é bastante perceptível para todas as temperaturas avaliadas em toda gama de conversão. Pode-se observar que o aquecimento por microondas aumentou as taxas iniciais de reação, resultando em uma reação mais rápida quando comparado com as taxas de reações para as reações com aquecimento convencional. De acordo com os valores obtidos, às taxas de reação para as reações com aquecimento convencional correspondem a $29 \%, 45 \%, 73 \%$ e $69 \%$ dos valores das taxas de reação utilizando radiação microondas, nas temperaturas de 50, 60, 70 e $80{ }^{\circ} \mathrm{C}$, respectivamente, para os primeiros 60 minutos de reação.

Conforme relatado por Costa et al. (2009), para polimerização em emulsão via radicais livres em reator microondas, a radiação microondas é preferencialmente absorvida pela água, devido suas características polares. Assim, a cinética da fase aquosa é afetada principalmente pela radiação microondas resultando em efeitos microondas específicos para cada sistema de monômeros. No caso da polimerização em miniemulsão apresentada neste trabalho, a radiação microondas pode acelerar a rotação das moléculas de monômero que contém os grupos funcionais ( $\mathrm{NCO}$ e $\mathrm{OH}$ ), aumentando a velocidade da reação. Além disso, o aquecimento convencional por convecção térmica através da parede do reator pode apresentar gradiente de temperatura no sistema de reação, assim, o efeito da falta de homogeneidade da temperatura pode levar a uma redução na velocidade da reação. O uso de radiação microondas, por outro lado, leva a um rápido aumento da temperatura diretamente relacionada ao movimento de rotação das moléculas possibilitando reações mais homogênea e rápida entre os grupos funcionais. Uma vez que a taxa de aquecimento e a eficiência do aquecimento dependem fortemente das propriedades dielétricas do reagente ou solvente, e em uma polimerização em emulsão ou miniemulsão 50 a 80 \% em massa do meio de reação é composta por água, a taxa de reação pode ser favoravelmente afetada pela radiação microondas favorecendo o aumento da taxa de reação.

Foi observada também a redução do diâmetro das nanopartículas com o tempo de reação para as reações em microondas para todas as temperaturas estudas, comportamento esse que não foi observado nas reações utilizando aquecimento convencional como apresentado nas Figuras $1 \mathrm{~b}$ e 1d. A redução do diâmetro das nanopartículas de PU sintetizadas a partir dos polióis óleo de mamona e PEG400 pode estar relacionada com o aumento da solubilidade dos monômeros na fase aquosa (e da água nas partículas), aumentado à formação de ureia e a liberação de $\mathrm{CO}_{2}$ que por sua vez estando presente nas nanopartículas ao evaporar provoca a redução do diâmetro das nanopartículas. Além da possível saída dos monômeros das partículas com o aumento do aquecimento devido ao aumento da mobilidade das cadeias.

\subsection{Efeito da potência máxima na polimerização em microondas}

O método dinâmico de operação do microondas é definido pelo ajuste de um único parâmetro cinético. Sendo assim estudou-se a influência da variação da potência máxima na faixa entre $50 \mathrm{~W}$ a $300 \mathrm{~W}$, mantendo a temperatura constante em $70{ }^{\circ} \mathrm{C}$ para reações utilizando óleo de mamona/PEG400 como poliol. Os resultados são apresentados na Tabela 3. 
Tabela 3 - Condições experimentais e resultados da polimerização em microondas utilizando diferentes potências máximas a temperatura constante $\left(70^{\circ}\right)$ na obtenção de PU.

\begin{tabular}{cccc}
$\begin{array}{c}\text { Potência máxima } \\
(\mathbf{W})\end{array}$ & Dp (nm) & PdI & $\begin{array}{c}\text { Taxa de reação aos 60 } \\
\text { min }(\% \mathbf{N C} / \mathbf{m i n})\end{array}$ \\
\hline 50 & $115 \pm 3$ & 0,126 & 0,717 \\
100 & $121 \pm 3$ & 0,129 & 0,724 \\
150 & $107 \pm 3$ & 0,131 & 1,064 \\
300 & $112 \pm 4$ & 0,127 & 1,267 \\
\hline
\end{tabular}

Como mostrado na Tabela 3 , os resultados indicam que o aumento da potência da radiação microondas não apresentou um efeito relevante no tamanho médio e o índice de polidispersão das nanopartículas de PU. Assim, sob as condições avaliadas, a potência da radiação microondas não apresentou influência na estabilidade da miniemulsão em termos de mecanismos de degradação, como a coalescência e maturação de Ostwald (degradação por difusão). Pela observação dos resultados é possível afirmar que a potência é um parâmetro cinético importante a ser controlado durante as reações de polimerização em miniemulsão na obtenção de PU em reator microondas. A radiação microondas atua no aumento da mobilidade das moléculas fazendo com que os grupamentos funcionais movam-se livremente ocasionando um aumento na velocidade de reação. Como apresentado na Tabela 3, quando a potência foi aumentada de $50 \mathrm{~W}$ para $300 \mathrm{~W}$ a velocidade da reação apresentou aumento de 0,717 para 1,267. Enquanto utilizando $50 \mathrm{~W}$ de potência foram necessários 240 min a $70{ }^{\circ} \mathrm{C}$ para alcançar $85 \%$ de conversão, utilizando $300 \mathrm{~W}$ a conversão alcançada foi de $97 \%$ em 60 min de reação.

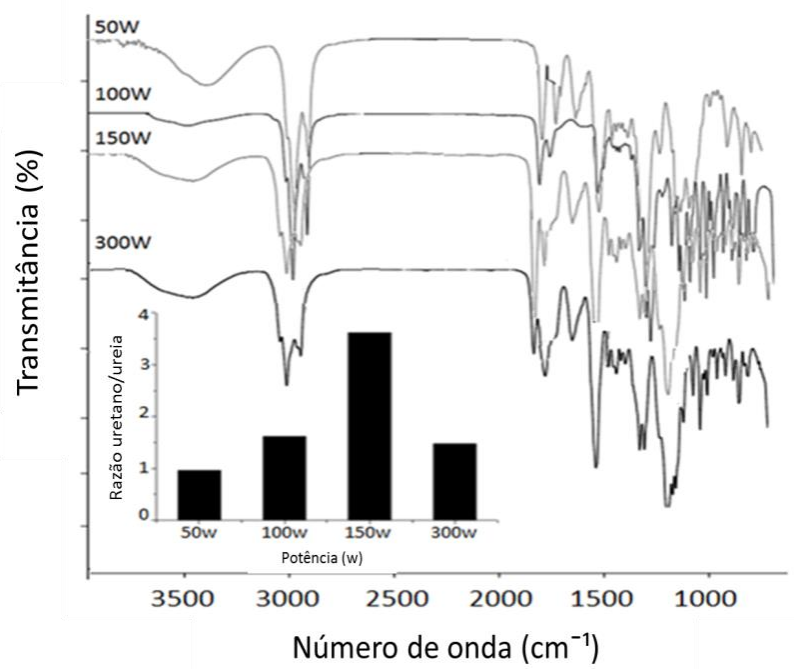

Figura 31 - Razão Uretano/ureia (inserção inferior à esquerda) calculado usando áreas de pico do FTIR para as amostras de PU sintetizadas via polimerização em miniemulsão em reator microondas à temperatura constante variando a intensidade da potência aplicada.

A partir dos espectros FTIR dos polímeros de PU foi possível obter a razão entre as áreas de uretano/ureia utilizando os picos localizados em $1680-1650 \mathrm{~cm}^{-1}$ para a ureia e 1740 - 
$1700 \mathrm{~cm}^{-1}$ para o uretano. Os resultados são ilustrados na Figura 3 onde, pode-se verificar que a formação de uretano aparece em um nível crescente com o aumento da potência na faixa entre 50 $\mathrm{W}$ e $150 \mathrm{~W}$, por outro lado quando a potência foi aumentada de $150 \mathrm{~W}$ para $300 \mathrm{~W}$ a razão uretano/ureia reduziu devido ao aumento na formação de ureia. O comportamento pode ser justificado pelo aumento da absorção da radiação microondas pelas moléculas de água aumentando assim a reação entre os grupamentos NCO livre com os grupamentos $\mathrm{OH}$ da água ocasionando um aumento na produção de ureia.

\section{CONCLUSÕES}

A utilização de radiação microondas acelerou as reações de polimerização na obtenção de poliuretano quando comparado com reações utilizando aquecimento convencional. Este fato foi atribuído à diferente solubilidade na fase aquosa e os parâmetros de dielétricos dos monômeros. Como consequência, os efeitos da radiação microondas são específicos para cada sistema. Um incremento significativo da taxa de reação foi observado nas reações com o aumento da potência máxima da radiação microondas. Na polimerização em etapas conduzida com até $300 \mathrm{~W}$ de potência máxima foi possível alcançar $97 \%$ de conversão em 60 minutos, enquanto que para a reação com potência máxima de $50 \mathrm{~W}$ foram necessárias cerca de 5 horas com aquecimento via microondas para alcançar a mesma faixa de conversão. Os resultados obtidos são relacionados com a propriedade de aquecimento seletivo da radiação microondas aumentando assim a interação entre os monômeros. A partir dos resultados apresentados neste trabalho é possível confirmar a vantagem da utilização de microondas também na cinética do processo de polimerização em etapas, quando comparado ao aquecimento convencional.

\section{REFERÊNCIAS}

ADLINGTON, K, G. JAOUAD, J. J.; HARFI, E.; DIMITRAKIS, G.; SMITH, A.; KINGMAN, S. W.; ROBINSON, J. P.; IRVINE, D. J. Mechanistic Investigation into the Accelerated Synthesis of Methacrylate Oligomers via the Application of Catalytic Chain Transfer Polymerization and Selective Microwave Heating. Macromolecules. v. 46, p. 3922-3930, 2013.

ASTM D-2572, Standard Test Method for isocyanate Groups in Urethane Materials or Prepolymers, (2003).

BOGDAL, D. Microwave-Assisted Polymerization. Polymer Sci: A Comprehensive Ref., v. 5, p. 981-1027, 2012.

COSTA, C.; SANTOS, A. F.; FORTUNY, M.; ARAUJO, P. H.H.; SAYER, C. Kinetic advantages of using microwaves in the emulsion polymerization of MMA. Mat. Sci. Eng. C., v. 29, p. 415-419, 2009. 


\section{9 a 22 de outubro de 2014 \\ Florianópolis/SC}

CRESPY, D.; STARK, M.; HOFFMANN-RICHTER, C.; ZIENER, U.; LANDFESTER, K. Polymeric Nanoreactors for Hydrophilic Reagents Synthesized by Interfacial Polycondensation on Miniemulsion Droplets. Macromolecules, v. 40, p.3122 - 31292007.

DING, M.; HE, X.; WANG, Z.; LI, J.; TAN, H.; DENG, H.; FU, Q.; GUC, Q. Cellular uptake of polyurethane nanocarriers mediated by gemini quaternary ammonium. Biomaterials., v. 32(35). p. 9515-9524, 2011.

FORTUNY, M.; RAMOS, A. L. D.; DARIVA, C.; EGUES, S. M. S.; SANTOS, A. F.; NELE, M.; COUTINHO, R. C. C. Principais aplicações das microondas na produção e refino de petróleo. Quim. Nova. v. 31, p.1553-1558, 2008.

GAUDIN, F.; SINTES-ZYDOWICZ, N. Poly(urethane-urea) nanocapsules prepared by interfacial step polymerization in miniemulsion. The droplet size: A key-factor for the molecular and thermal characteristics of the polymeric membrane of the nanocapsules?. Col. Surf. A: Phys. Eng. Asp., v, 384, 698-712, 2011.

MARCASUZA, P.; REYNAUD, S.; GRASSL, B.; PREUD’HOMME, H.; DESBRIÈRES, J.; TRCHOVÀ, M.; DONARD, O. F. X. Microwave synthesis: An alternative approach to synthesize conducting end-capped polymers. Polymer. v. 52, p. 33-41. 2011.

MELO, C. A. R. J.; ALBUQUERQUE, C. R.; CARNEIRO, J. S. A.; DARIVA, C.; FORTUNY, M.; SANTOS, A. F.; EGUES, S. M. S.; RAMOS, A. L. D. Solid-Acid-Catalyzed Esterification of Oleic Acid Assisted by Microwave Heating. Ind. Eng. Chem. Res. v. 49 (23), p.12135-12139, 2010.

TORINI, L.; ARGILLIER, J. F. AND ZYDOWICZ, N. Interfacial Polycondensation Encapsulation in Miniemulsion. Macromolecules. v.38, p. 3225-3236, 2005.

VALERIO, A.; ARAUJO, P. H. H.; SAYER, C. Preparation of poly(urethane-urea) nanoparticles containing açaí oil by miniemulsion polymerization. Polímeros. v. 23, p. 451-455, 2013.

ZANETTI-RAMOS, B. G.; LEMOS-SENNA, E.; CRAMAIL H; CLOUTET, E; BORSALI, R.; SOLDI, V. The role of surfactant in the miniemulsion polymerization of biodegradable polyurethane nanoparticles. Mat. Sci. Eng. C. v. 28, 526- 531, 2008.

XIONG, S.; GUO, X.; LI, L.; WU, S.; CHU, P. K.; XU, Z. Preparation and characterization of fluorinated acrylate copolymer latexes by miniemulsion polymerization under microwave irradiation. J. Fluorine Chem. v. 131, 417-425, 2010.

YU, Y.-Y.; P.-K., Chen. Nanocomposites of polymer and inorganic nanoparticles prepared by focused microwave polymerization for optical thin films applications. Thin Solid Films. v. 544, p. 48-53, 2013. 